\title{
La mejora de la calidad de los servicios a través de su medición
}

(1) Ma Eva Diz Comesaña

(2) Nuria Rodríguez López

\section{RESUMEN}

Las características de intangibilidad, heterogeneidad y simultáneidad de producción y consumo de los servicios hacen que la gestión de la calidad sea un tema complejo en este sector. Así lo demuestra la diversidad existente en la literatura académica tanto en el concepto y componentes de calidad como en las herramientas de medidas asociadas.

En este trabajo se pretende abordar la gestión de la calidad desde la perspectiva de las empresas de servicios, con la finalidad de sistematizar los distintos componentes y poder mejorar los resultados relativos a esta prioridad competitiva a través de su medición. En lo que se refiere al concepto y componentes de la calidad se analizarán las diferencias entre calidad objetiva y subjetiva. En cuanto a la medición de la calidad, se abordarán las principales medidas clasificándolas en internas, externas e híbridas.

Palabras clave: Sector servicios, calidad, costes de calidad, medición de la calidad.

THE IMPROVEMENT OF THE SERVICES QUALITY THROUGH ITS MEASUREMENT

\section{ABSTRACT}

The services are products characterized for their intangibility, heterogeneity and simultaneous occurrence of production and consumption. Because of these characteristics, the services qualitiy management become a extremely complex issue. This fact can be demostrated by the diverse academic literature existent about the subject.

In this paper we analyze the quality management from the service firm view, with the aim of deepening the concept of quality, its components and how they can improve the organizational outcomes concerning this competitive priority through its measurement. In regard to the concept and quality components we focus on the differences between objective and subjective quality. As far as quality measure, we address the main measures classified into internal, external and hybrid.

Keywords: Services sector, quality, quality costs, quality measures.

\section{INTRODUCCIÓN}

Aproximadamente en los años ochenta del siglo pasado es cuando la calidad comienza a ser considerada como una variable estratégica, por tanto, capaz de proporcionar una ventaja competitiva a la empresa. Es también en esta época cuando surge el afán por la mejora de la calidad en los servicios. Ante esta situación, las empresas de servicios se encuentran con la difícil tarea de trasladar los principios de mejora de la calidad del sector manufacturero al terciario, siempre que esto sea factible, ya que ambos disponen de características distintas. En concreto, bajo este contexto podrían destacarse fundamentalmente la intangibilidad, la heterogeneidad, el carácter perecedero y la simultaneidad de la producción y el consumo, como características definitorias de los servicios (Zeithaml et al., 1985; Rushton y Carson, 1989).

De más está decir que estas características no las poseen todos los servicios al mismo nivel. En realidad, lo que existe es un continuum en las mismas y los servicios disponen de una mayor tendencia hacia ellas (Payne, 1993).

La confluencia de estas características proporciona ciertos problemas para la mejora de la calidad en el sector servicios, entre los que destacan los siguientes (véase Figura 1):

La medida de calidad ofrecida a los clientes de la empresa.- La dificultad que supone la medida de la calidad está directamente relacionada con la intangibilidad, el carácter perecedero y la heterogeneidad.

El protagonismo que va a tener el personal en contacto.- La heterogeneidad y la simultaneidad de la producción y el consumo hacen que tenga lugar una interacción directa entre el cliente y el productor del servicio, por lo que el empleado que atiende al cliente toma un mayor protagonismo. 


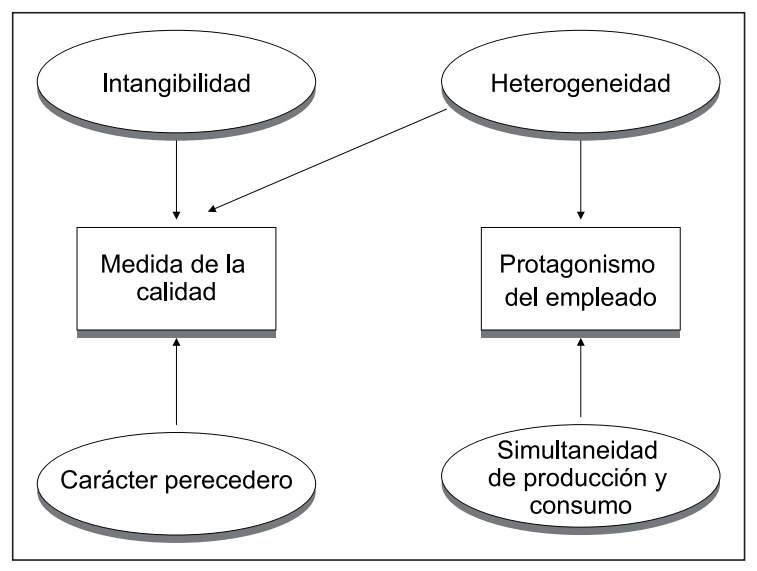

Fuente: Fernández (1996, p. 114).

Figura 1. Características distintivas de los servicios y problemática peculiar que plantean para la mejora de la calidad.

En los siguientes epígrafes se pretende profundizar en el primero de estos aspectos, abordando en primer lugar el concepto de calidad y los distintos componentes de la calidad en las empresas de servicios, para pasar a analizar, posteriormente, los resultados en torno a esta prioridad competitiva, centrando especial atención en la búsqueda de instrumentos que permitan a la empresa medir la calidad de los servicios ofertados a los clientes, de cara a conseguir su mejora.

\section{EL CONCEPTO DE CALIDAD Y SUS COMPO- NENTES}

En la sociedad actual existe una gran variedad de bienes -tangibles e intangibles- capaces de satisfacer casi cualquier demanda que se genere. Ante esta situación, el cliente elegirá aquel producto y/o servicio, que estando dentro del rango de precios que se haya marcado, le resulte más adecuado. Precisamente el concepto de calidad que tiene el consumidor, consiste en la adecuación e idoneidad al uso. Sin embargo, para la empresa esta definición resulta insuficiente, necesita algo más tangible.

Numerosos investigadores han hecho referencia al concepto de calidad ${ }^{3}$, por lo que existen distintas acepciones además de diferentes tipologías. Así, por ejemplo, Grönroos (1990) clasifica la calidad en: calidad funcional y calidad técnica, refiriéndose al proceso de servicio y al resultado real del mismo, respectivamente. Lutz (1986) distingue

3 Diversos autores han realizado una revisión de los principales estudios sobre calidad. Véase: Ahire et al. (1995), Álvarez et al. (2000), Calvo de Mora et al. (2003), Molina et al. (2003), Del Río y Martínez (2007). entre calidad afectiva, vinculada a los bienes intangibles, y calidad cognoscitiva, ligada a bienes de consumo durables; este segundo tipo suele estar asociado a situaciones en las que la cantidad de atributos que se pueden evaluar antes de la adquisición es mayor a aquéllos que solamente se pueden valorar durante el consumo. $Y$ distintos autores, tales como Garvin (1984), Holbrook y Corfman (1985), Parasuraman et al. (1988), Zeithaml (1988), Cruz y Múgica (1993), Requena (2008), han tratado de enfatizar la diferencia entre calidad objetiva y percibida.

Dada la gran importancia de esta última clasificación, en este trabajo se cree conveniente abordar este tema comenzando por tratar de aclarar las diferencias entre calidad percibida y calidad objetiva.

En este sentido, Garvin (1984) describe tres maneras de definir la calidad según se base en el producto, en la producción o en el uso. La calidad basada en el producto se refiere a los atributos o componentes del mismo; la calidad basada en la producción consiste en el nivel de concordancia de los resultados con las especificaciones propuestas $^{4} ; \mathrm{y}$ la calidad basada en el uso hace referencia a la calidad percibida.

Los dos primeros tipos, basados en producto y producción, se corresponden con la calidad objetiva. A este respecto, autores, como Curry y Faulds (1986), describen la calidad objetiva como la superioridad cuantificable y verificable de algún/os estándar/es ideal/es predeterminado/s, aunque en los trabajos que defienden esta postura no ha habido acuerdo sobre los estándares a emplear. En estos casos, la calidad objetiva es entendida como superioridad técnica o excelencia de los productos.

El tercer tipo, basado en el uso se corresponde con la definición de calidad percibida de Zeitham (1988), es decir, la valoración que el consumidor realiza sobre la superioridad del servicio ${ }^{5}$, de modo que puede ser considerada como una modalidad de actitud. Siguiendo esta línea, otros autores, como Grönroos (1990), sugieren que la calidad percibida de un servicio resulta de un proceso de evaluación, en el que los clientes comparan sus percepciones

4 La filosofía japonesa cero defectos es una de las técnicas que permiten hace referencia a la calidad basada en la producción (Reichheld y Sasser, 1992).

5 La calidad percibida no equivale a la satisfacción -aunque están íntimamente relacionadas-, obteniéndose esta última a través de la confrontación de expectativas y percepciones (Teas, 1993; Parasuraman et al., 1994; Vázquez, Díaz y Rodríguez, 1997). La relación entre estos dos conceptos no está del todo clara, autores como Bitner (1990) y Bolton y Drew (1991) indican que la satisfacción es una antecedente a la calidad percibida. Otros como Parasuraman et al. (1985-1988), Cronin y Taylor (1992), Anderson et al., (1994) opinan lo contrario, que la calidad percibida es un antecedente de la satisfacción. Otros, como Oliver (1980), indican que la satisfacción permite pasar de las percepciones a priori sobre la calidad a la formación de las nuevas percepciones. 
de la prestación del servicio y su resultado con lo que ellos esperaban.

Autores como Vázquez, Díaz y Rodríguez (1997, pp. 120-121) tratando de definir el concepto de cali- dad, también distinguen entre calidad objetiva y calidad subjetiva (o percibida), recogiendo una serie de características diferenciadoras para cada tipo (véase cuadro 1).

Cuadro 1. Aproximación al concepto de calidad.

\begin{tabular}{|c|c|}
\hline Calidad objetiva & Calidad subjetiva \\
\hline $\begin{array}{l}\text { - } \quad \text { Visión interna de la calidad. } \\
\text { - } \quad \text { Adaptación a especificaciones preestablecidas } \\
\text { - } \quad \text { Prestación sin errores, reduciendo costes y } \\
\text { evitando desviaciones respecto al estándar } \\
\text { establecido. } \\
\text { - Adecuada para actividades estandarizadas. }\end{array}$ & $\begin{array}{l}\text { - } \quad \text { Visión externa de la calidad. } \\
\text { - } \quad \text { Enfoque de marketing-demanda. } \\
\text { - } \quad \text { Habilidad de la empresa para determinar las ne- } \\
\text { cesidades, deseos y expectativas de los clientes. } \\
\text { - } \quad \text { Actividades de elevado contacto con clientes. }\end{array}$ \\
\hline
\end{tabular}

Fuente: Vázquez, R.; Díaz, A.M. Y Rodríguez, I.A. (1997, p. 120).

Holbrook y Corfman (1985, p. 33) utilizando otras denominaciones realizan una división similar, ellos distinguen entre calidad mecánica y calidad humana. La primera se refiere al aspecto objetivo o distintivo de una cosa o suceso. Y la calidad humana es la relativa a la respuesta subjetiva de la gente.

Sin embargo, es interesante resaltar que no todos los autores apoyan esta clasificación. Así, por ejemplo, Garvin (1984) precisa que aunque los conceptos de calidad objetiva y superioridad técnica de un producto están relacionados, no se trata de lo mismo. Además, los conceptos relativos a la calidad objetiva en cierta medida también se basan en percepciones, ya que aunque se trabaje con medidas reales, las especificaciones fijadas dependen de lo que los directivos consideren oportuno.

Asimismo, Holbrook y Corfman (1985, p. 33), al igual que Olshavski (1985), proponen que la calidad es una forma de evaluar o valorar un producto o servicio, siendo a menudo similar a una actitud. Incluso investigadores, como Maynes (1976), consideran que la calidad es subjetiva por naturaleza y que la calidad objetiva no existe. También Zeithaml (1988, p.3), sin afirmar totalmente que la calidad percibida es distinta de la objetiva, indica que esta última puede que no exista ya que toda calidad es percibida por alguien. En concreto, otros investigadores como Parasuraman et al. (1985-1988), Bolton y Drew (1991) y Cronin y Taylor (1992) apoyan esta postura cuando abordan el caso de los servicios.

Con el objetivo de incorporar distintas perspectivas, Garvin (1988) identificó una serie de componentes relativos a la calidad, que podrían resultar útiles como marco de referencia para el análisis y la planificación estratégica, éstos son:

1. Desempeño: Características operacionales principales.

2. Características: Aspectos que llaman la atención.

3. Confiabilidad: Probabilidad de un mal funcionamiento o de un fallo.

4. Conformidad: Habilidad para cumplir con las especificaciones.

5. Durabilidad: Durante cuánto tiempo el producto seguirá proporcionando un valor al cliente.

6. Aspectos del servicio: Rapidez, cortesía, competencia y facilidad para corregir los problemas.

7. Estética: De qué forma el producto atrae cualquiera de los cinco sentidos del cliente.

8. Calidad percibida ${ }^{6}$ : Asociaciones con la reputación de la compañía o el nombre de la marca.

Estos componentes son desarrollados desde la perspectiva de las manufacturas, aunque pueden tener cierta aplicación en los servicios. No obstante, la mayoría de los investigadores consideran que ante las características diferenciadoras de los bienes intangibles, éstos requieren un enfoque distinto.

Las investigaciones más extensas sobre la calidad del servicio están orientadas al usuario. En ese sentido, Zeithaml et al. (1990) identificaron una

6 Garvin utiliza el término de calidad percibida no con la misma amplitud con la que fue tratado por otros autores, tal como se ha visto en el apartado anterior. 
serie de criterios que utilizan los consumidores para evaluar la calidad del servicio, éstos son los siguientes ${ }^{7}$

1. Credibilidad: Integridad, credibilidad y honestidad del proveedor del servicio.

2. Seguridad: Libre de peligros, riesgos o dudas.

3. Acceso: Accesibilidad y facilidad de contacto.

4. Comunicación: Escuchar a los clientes y mantenerlos informados, empleando un lenguaje que puedan comprender.

5. Comprensión del cliente: Hacer un esfuerzo para conocer a los clientes y saber cuáles son sus necesidades.

6. Tangibles: Apariencia de las instalaciones físicas, el equipo, el personal y los materiales de comunicación.

7. Confiabilidad: La habilidad de desempeñar el servicio prometido en una forma confiable y precisa.

8. Actitud abierta: Una buena disposición para ayudar a los clientes y proporcionarles un servicio rápido.

9. Competencia: La posesión de las habilidades y el conocimiento requeridos para el desempeño del servicio.

10. Cortesía: Educación, respeto, consideración y actitud amistosa del personal que tiene contacto con el cliente.

En investigaciones posteriores, estos mismos autores (Berry et al., 1992) encontraron un alto grado de correlación entre algunas de estas variables, de tal forma que estas diez dimensiones se concretaron en cinco:

1. Tangibles: Apariencia de los elementos físicos.

2. Confiabilidad: Un desempeño confiable y preciso.

3. Actitud responsiva: Prontitud y espíritu servicial.

4. Garantía: Competencia, cortesía, credibilidad y seguridad.

5. Empatía: Acceso fácil, buenas comunicaciones y comprensión del cliente.

Estas dimensiones son las utilizadas en el cuestionario SERVQUAL ${ }^{8}$

7 Han aparecido también otros trabajos sobre el tema con enfoques distintos, algunos de ellos (Cronin y Taylor, 1992; Teas, 1993) manifestando ciertas críticas sobre el primero.

8 El cuestionario SERVQUAL utilizando las diez dimensiones indicadas en primer lugar puede verse en: Parasuraman et al., 1988. Utilizando las cinco dimensiones propuestas posteriormente puede verse en: Parasuraman et al., 1991.
El conocer qué es lo que esperan los consumidores es, por supuesto, parte del reto. Otra parte -una gran parte- es encontrar estas expectativas o esperanzas. Berry et al. (1992) se hacen una pregunta fundamental: ¿Qué servicios deben interesarle mejorar a cada empresa para superarse? Y responden con cinco cuestiones:

1. Definir el rol del servicio.

2. Competir por talento y hacer uso del mismo.

3. Poner énfasis en los equipos de trabajo.

4. Búsqueda de la fiabilidad.

5. Ser hábiles ante la resolución de problemas.

Las dimensiones tratadas junto con estas cuestiones ponen de manifiesto la importancia del personal en contacto (Singh, 2000). Según Drummond (1995, p. 167), el personal en contacto "desempeña un papel fundamental en la prestación del servicio porque:

1. Esos empleados representan a la empresa.

2. Son los primeros en enfrentarse a la crisis.

3. Se encargan de la potencialmente volátil interfaz con el cliente."

No obstante, con la automatización de los servicios, el personal en contacto suele perder importancia, lo que podría indicar un menor nivel de calidad. Sin embargo, aunque hay ciertas dimensiones que pueden resultar perjudicadas (cortesía, comunicación...), puede que otras encuentren en la automatización la forma de mejorar (precisión, prontitud...). Ante esta situación, es labor de la dirección el saber combinar los recursos humanos y tecnología pertinente para alcanzar el nivel de calidad adecuado.

\section{MEDICIÓN Y MEJORA DE LA CALIDAD EN LOS SERVICIOS}

Según Pérez y Parra (2007), la evaluación y análisis de la calidad es el primer paso para avanzar hacia la confiabilidad, entendida ésta en una forma práctica, como la calidad a través del tiempo (Escobar et al., 2003, p. 5-6). En este sentido, la medición de la calidad resulta de vital importancia, pudiendo considerarse como la comparación entre la calidad real y la calidad propuesta o esperada, cuando la primera es superada por la segunda, su mejora pasa por actuar sobre dicha diferencia (véase Figura 2) 


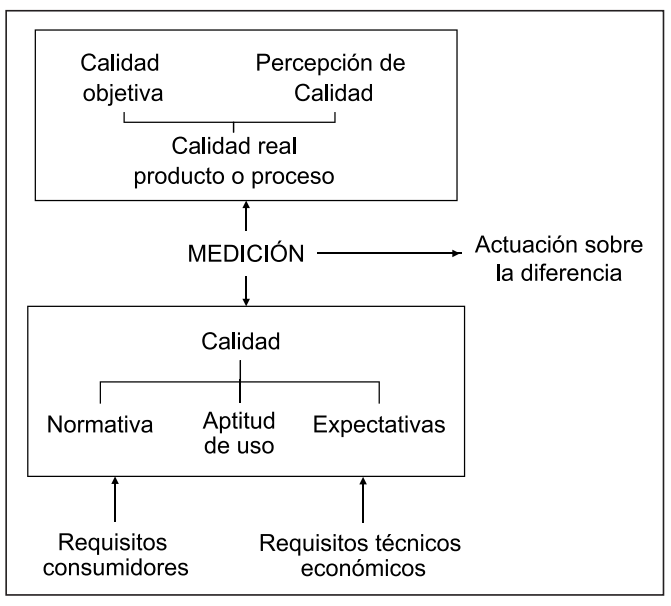

Fuente: Murillo y Saurina (p. 636).

Figura 2. Medición de la calidad en la prestación de servicios.

Dentro de las medidas de calidad que utilizan las empresas pueden establecerse dos grupos: internas y externas (Bolton y Drew, 1994) (véase figura 3). Las primeras son utilizadas tradicionalmente por las propias empresas para valorar la calidad y consisten en la comprobación del cumplimiento de ciertos estándares prefijados.

Dentro de las medidas internas de la calidad se puede encuadrar el grupo de técnicas relativas al control estadístico (Price, 1984; Deming, 1989). Éstas se basan en la comparación del diseño del servicio con su posterior concreción, enfocándose más en el proceso que en el output -difícilmente inspeccionable en muchos servicios-. Las principales herramientas de control estadístico aplicables a los bienes intangibles son: el diagrama de flujo de servicio, los gráficos de control de proceso, el diagrama causa-efecto, el diagrama de Pareto, etc. (Rosander, 1992, pp. 159-204, 523-578).

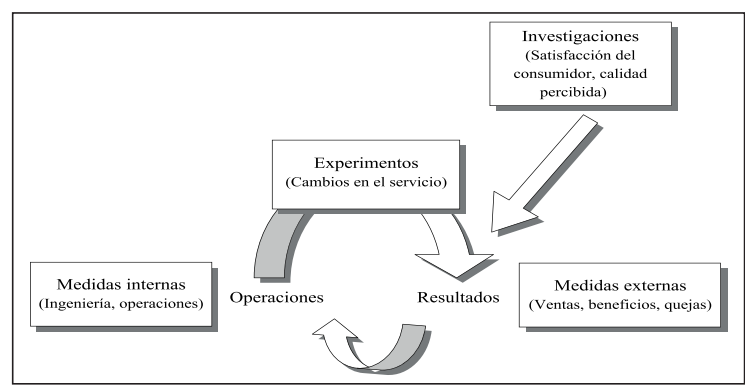

Fuente: Bolton y Drew (1994, p. 175).

Figura 3. Ciclo de satisfacción del cliente.
Respecto a las medidas externas, éstas son originadas a partir de los clientes ${ }^{9}$, y se podrían destacar: el análisis de los clientes que abandonan la empresa y la investigación de las quejas realizadas por los consumidores, tratando de aprovechar estas últimas para estrechar los lazos con los mismos.

En cuanto al análisis de los clientes que abandonan la empresa hay que decir que, salvo excepciones, se trata de clientes insatisfechos, por tanto, se trata de un indicador de la mala calidad del servicio.

Por lo que se refiere a las quejas (Tschohl y Franzmeier, 1994; Fernández, 1996, pp. 120-123) realizadas por los usuarios del servicio, en principio, menos quejas parece indicar una mayor calidad, sin embargo, en ocasiones, la no presencia de quejas no indica que los clientes estén satisfechos, sino que se van sin protestar porque perciben que hacerlo no sirve de nada (Keiser, 1988). Este aspecto es de especial atención si se tiene en cuenta que los clientes transmiten más sus experiencias con servicios deficientes que las relacionadas con servicios satisfactorios (Ivancevich et al., 1996).

Aún con todo, no cabe duda de que las quejas realizadas a la propia empresa pueden convertirse en una oportunidad para la misma si son bien tratadas, por lo que puede resultar interesante el facilitar la prestación de las mismas.

En concreto, el incremento o disminución del número de quejas suele ser bastante representativo para analizar la evolución de la calidad cuando se produce algún cambio en la empresa que afecta a la prestación del servicio, ya que si la calidad mejora el número de quejas suele disminuir salvo que antes este fuese nulo, caso en que suele incrementarse el número de elogios, los cuales suelen ser considerados por el proveedor como un menor número de quejas. En el caso de que la calidad empeore, suele tener lugar un incremento en el número de quejas, ya que si el cliente estaba más a gusto con la situación anterior, éste suele manifestarse porque esto puede servir para volver a dicha situación, y además aunque el cliente considere apropiado un cambio de proveedor, la queja no deja de ser una forma de reivindicar su posición.

Ante estas medidas hay que indicar que la investigación de mercados juega un importante papel, porque puede aportar información relevante como medida externa de la calidad de los servicios, de hecho, con la aparición de la Gestión de la Calidad

9 Los datos de la encuesta Gallup realizada por al American Society fo Quality Control (ACQC) en 1985 ponen de manifiesto que los clientes también son capaces de disponer medidas de la calidad de los servicios (Rosander, 1992, pp. 52-59). 
Total (Oakland, 1989; Atkinson, 1990) (TQM), el énfasis ha evolucionado hacia la satisfacción del consumidor, pasando a usar técnicas relacionadas con la investigación de mercados (Garvin, 1991). En este sentido, pueden destacarse el modelo SERVQUAL (Parasuraman et al., 1988-1991), el modelo SERVPERF (Cronin y Taylor, 1992-1994) y el modelo del desempeño evaluado (Teas, 1993) -estos dos últimos desarrollados a partir del primero-, etc.

Por otro lado, los costes de la calidad son otra medida que se puede utilizar en el caso de los servicios, esta se encuentra a medio camino entre las internas y externas, aunque suele ser incluida dentro de las primeras.

Los costes de la calidad en los servicios están compuestos por (Crosby, 1989; Campanella, 1992 Carr, 1992; Groocock, 1993; Barnes y Cumby, 1996; Fernández, 1996):

1. Costes de conformidad: Éstos engloban los costes de prevención y evaluación.

Los costes de prevención abarcan todas aquellas actividades diseñadas para evitar que haya defectos en los servicios a suministrar, tales como los costes de la planificación de la calidad, los costes de la calidad de muestras y/o piezas y los costes de la formación de trabajadores.

Los costes de evaluación son los relativos a la medida, evaluación o auditoria de servicios para poder garantizar la adecuación a los estándares de calidad y requisitos de funcionamiento.

2. Coste de no conformidad o costes de fallo por no cumplir con las exigencias del cliente. Estos costes son los que resultan de evaluar y corregir o sustituir aquellos servicios que no se adaptan a las necesidades o requisitos del cliente. Dentro de estos costes se pueden distinguir dos subtipos:

Costes de fallo interno: son aquellos que se producen antes de la distribución del servicio, tales como los costes de reparación y de revisión.

Costes de fallo externo: son aquellos que se producen tras la entrega del servicio al cliente, tales como los relativos a las quejas de los clientes, las devoluciones, los servicios postventa y las garantías.

3. Costes por las oportunidades perdidas ${ }^{10}$ : Estos costes son los relativos a los clientes perdidos y la pérdida de ingresos futuros asociados.

10 La inclusión de los costes por las oportunidades perdidas hace que esta medida se encuentre a medio camino entre las medidas internas y externas de la calidad del servicio
Normalmente, un incremento de los costes de prevención será más que compensado por la disminución de los costes de no conformidad y de los costes por oportunidades perdidas. La estrategia a seguir para los costes de la calidad consiste pues en tratar de minimizar los costes de fallos e invertir en las actividades relativas a la prevención, y así poder reducir también los costes de evaluación.

\section{CONCLUSIÓN}

La calidad se manifiesta como una prioridad competitiva un tanto subjetiva, subjetividad que se ve incrementada al referirse al sector servicios. Ante esta situación, resulta fundamental identificar una serie de componentes relativos a la calidad que actúen como marco de referencia para la mejora de la misma. No obstante, los servicios poseen una serie de características que dificultan la medición de la calidad y que confieren gran protagonismo al personal de contacto. Es por ello, que se ha creído conveniente analizar las principales medidas de la calidad de los servicios, distinguiendo entre medidas internas y externas. Dentro de las primeras se ha recogido el control estadístico de la calidad, proponiendo herramientas como el el diagrama de flujo de servicio, los gráficos de control de proceso, el diagrama causa-efecto, el diagrama de Pareto, etc. $Y$ dentro de las segundas se han destacado el análisis de los clientes que abandonan la empresa y la investigación de las quejas realizadas por los consumidores, donde la investigación de mercado juega un papel importante. Además, se han analizado los costes de la calidad como una medida de carácter interno-externo. Todas estas medidas ofrecen la oportunidad de conocer mejor el nivel de calidad, para poder mejorarlo a lo largo del tiempo.

\section{REFERENCIAS BIBLIOGRÁFICAS}

[1] Ahire, A.L.; Landeros, R. y Colhar, D.Y. (1995): "Total Quality Management: A literature Review and a Agenda for Future Research", Production and Operations Management, Vol. 4, N. ${ }^{\circ} 3$, pp. 227-306.

[2] Álvarez, M.J.; Montes, M. y Peña, D. (2000): "Total Quality Management: A Literature Review and Future Research Directions for the XXI Century", POMS Sevilla, Select Papers, pp. 279-289.

[3] Anderson, E.W.; Fornell, C. y Lehmann, D.R (1994): "Customer Satisfaction Market Share, and Profitability: Findings form Sweden", Journal of Marketing, Vol. 58, pp. 53-66. 
[4] Atkinson (1990): Creating Culture Change: The Key to Successful Total Quality Management, IFS Publications.

[5] Barnes, J.G. y Cumby, J.A. (1996): "The Cost of Service Quality: Extending the Boundaries of Accounting Systems to Enhance Customer Value", en GLYNN, W.J. y BARNES, J.G. (eds.): Understanding Services Management, John Wiley \& Sons Ltd, Chichester, Inglaterra, pp. 178-202.

[6] Berry, L.L.; Zeithaml, V.A. y Parasuraman, A. (1992): "Five Imperatives for Improving Service Quality”, en LOVELOCK, C.H.: Managing Services. Marketing, Operations, and Human Resources, Prentice-Hall, Londres, pp. 224-235.

[7] Bitner, M.J. (1990): "Evaluating Service Encounters: The Effects of Physical Surroundings and Employee Rerponses", Journal of Marketing, Vol. 54, pp. 69-82.

[8] Bolton, R.N. y Drew, J.H. (1991): “A Multistage Model of Customer's Assessments of Service Quality and Value", Journal of Consumer Research, Vol. 17, pp. 375-384.

[9] Bolton, R.N. y Drew, J.H. (1994): "Linking Customer Satisfaction to Service Operations and Outcomes", en RUST, R.T. y OLIVER, R.L. (eds.): Service Quality: New Directions in Theory and Practice, Sage, California.

[10] Calvo de Mora, A.C.; Criado, F. y Pizarro, M.I. (2003): "Situación y perspectiva para el siglo XXI de la investigación empírica en el ámbito de la gestión y mejora de la calidad", Revista Europea de Dirección y Economía de la Empresa, Vol. 12, N. ${ }^{\circ} 1$, pp. 43-68.

[11] Campanella, J. (1992): Principios de los costes de la calidad, Madrid: Díaz de Santos.

[12] Carr, L.P. (1992): “Applying Cost of Quality to a Service Business", Sloan Management Review, Verano, pp. 72-77.

[13] Cronin, J.J. y Taylor, S.A. (1992): "Measuring Service Quality: A Reexamination and Extension", Journal of Marketing, Vol. 56, Julio, pp. 55-68.

[14] Cronin, J.J. y Taylor, S.A. (1994): "SERVPERF Versus SERVQUAL: Reconciling PerformanceBased and Perceptions-Minus-Expectations Measurement of Service Quality", Journal of Marketing, Vol. 58, enero, pp. 125-131.

[15] Crosby, P.B. (1989): La calidad no cuesta, México: Compañía Editorial Continental.
[16] Cruz, I, y Múgica, J.M. (1993): “La relación precio-calidad objetiva en los mercados de productos de gran consumo", Información Comercial Española. Revista de Economía, N. ${ }^{\circ} 716$, abril, pp. 25-35.

[17] Curry, D.J. y Faulds, D.J. (1986): "Indexing Product Quality: Issues, Theory, and Results", Journal of Marketing, Vol. 13, Junio.

[18] Del Río, M.C. y Martínez, M. (2007): “Tendencias de la investigación empírica en el ámbito de la gestión de la calidad", Investigaciones Europeas de Dirección y Economía de la Empresa, Vol. 13, N. ${ }^{\circ}$ 1, pp. 91-102.

[19] Deming, W.E. (1989): Calidad, Productividad y Competitividad, Madrid: Díaz de Santos.

[20] Drummond, H. (1995): Qué es hoy la Calidad Total. El movimiento de la calidad, Barcelona: Ediciones Deusto.

[21] Escobar, L.A.; Villa, E.R. y Yáñez, S. (2003): "Confiabilidad:Historia, estado del arte y desafíos futuros", DYNA, Noviembre, Vol. 70, N. ${ }^{\circ}$ 140 , pp. $5-21$.

[22] Fernández, M. (1996): "Cómo medir la calidad en los servicios", ICE Tribuna de Economía, N. ${ }^{\circ}$ 755, pp. 113-125.

[24] Garvin (1991): "How the Baldrige Award Really Works", Harvard Business Review, NoviembreDiciembre, pp. 80-93.

[25] Garvin, D.A. (1984): "La Calidad en la Línea de Producción", Harvard-Deusto Business Review, $2 .^{\circ}$ trimestre.

[26] Garvin, D.A. (1988): Managing Quality, Nueva York: The Free Press.

[27] Grönroos, C. (1990): Service Management and Marketing, Lexington, MA: Lexigton Books.

[28] Groocock, J.M. (1993): La cadena de la calidad, Madrid: Díaz de Santos.

[29] Holbrook, M.B. y Corfman, K.P. (1985): “Quality and Value in the Consumption Experience: Phaedrus Rides Again", en JACOBY, J. y OLSON, J.C. (eds.): Perceived Quality, Lexington, MA: Lexington Books.

[30] Ivancevich, J.M.; Lorenzi, P. y Skinner, S.J. (1996): Gestión Calidad y Competitividad, Irwin, Madrid, pp. 621.

[31] Lutz, R. (1986): "Quality is as Quality Does: An Attitudinal Perspective on Consumer Quality Judgments", Presentation to the Marketing Science Institute Trustees, Meeting, Cambridge, MA. 
[32] Maynes, E.S. (1976): "The Concept and Measurement of Product Quality", Household Production and Consumetions, N. ${ }^{\circ} 40$.

[33] Molina, L.M.; Lloréns, F.J. y Fuentes, M.M (2003): "La investigación en gestión de la calidad en España (1990-1999)", Cuadernos de Economía y Dirección de la Empresa", N. ${ }^{\circ} 16$, pp. 5-26.

[34] Murillo, C. y Saurina, C.: "Medición de la calidad en la prestación de servicios personales", IX Reunión ASEPELT España, pp. 631-643

[35] Oakland (1989): Total Quality Management, Butterworth Heinneman.

[36] Oliver, R.L. (1980): "A Cognitive Model of the Antecedents and Consequences of Satisfaction Decision", Journal of Marketing Research, Vol. 17, pp. 460-469.

[37] Olshavsky, R.W. (1985): "Perceived Quality in Consumer Decision Making: An Integrated Theoretical Perspective", en JACOBY, J. y OLSON, J.C. (eds.): Perceived Quality, Lexington Books, Lexington, MA.

[38] Parasuraman, A.; Zeithalm, V.A. y Berry, L.L. (1985): "A Conceptual Model of Service Quality and its Implications for Future Research", Journal of Marketing, Vol. 49, Otoño.

[39] Parasuraman, A.; Zeithalm, V.A. y Berry, L. (1988): "SERVQUAL: A Multiple-Item Scale for Measuring Consumer Perceptions of Service Quality", Journal of retailing, Vol. 64, N. ${ }^{\circ} 1$, pp. 12-40.

[40] Parasuraman, A.; Zeithalm, V.A. y Berry, L.L. (1991): "Refinement and reassessment of the SERVQUAL scale", Journal of Retailing, Vol. 67, N. $^{\circ} 4$, pp. $420-450$.

[41] Parasuraman, A.; Zeithaml, V.A. y Berry, L.L. (1994): "Reassessment of Expectations as a Comparison Standard in Measuring Service Quality: Implications for Further Research", Journal of Marketing, Vol. 58, enero, pp. 111124.

[42] Payne, A. (1993): The Essence of Services Marketing, Londres: Prentice-Hall.

[43] Pérez, J. y Parra, C. (2007): "Evaluación y análisis de la calidad de un servicio de apoyo desde la perspectiva del usuario: primer paso hacia la confiabilidad", Industrial Data, enero/ junio, Vol.10, N. ${ }^{\circ} 1$, pp. 70-79.

[44] Price, F. (1984): Right first time, Gower, Aldershot.

[45] Reichheld, F.F. y Sasser, W.E. (1992): "Zero Defections: Quality Comes to Services", en Lovelock, C.H.: Managing Services. Marketing, Operations, and Human Resources, Londres: Prentice-Hall, pp. 250-258.

[46] Requena, M. (2008): El desarrollo de las marcas gestionadas por la distribución. Análisis de las variables relevantes, Tesis doctoral.

[47] Rosander, A.C. (1992): La búsqueda de la calidad en los servicios, Díaz de Santos, Madrid, pp. 52-59.

[48] Rushton, A.M. y Carson, D.J. (1989): “The Marketing of Services: Managing the Intangibles", European Journal of Marketing, Vol. 23, N. ${ }^{\circ} 8$, pp. 23-44.

[49] Singh, J. (2000): "Performance Productivity and Quality of Frontline Employees in Service Organizations", Journal of Marketing, Vol. 64, abril, pp. 15-34.

[50] Teas, R.K. (1993): "Expectations, Performance, Evaluation and Consumer's Perceptions of Quality", Journal of Marketing, Vol. 57, octubre, pp. 18-34.

[51] Tschohl, J. y Franzmeier, S. (1994): Alcanzando la excelencia mediante el servicio al cliente, Madrid: Díaz de Santos, pp. 325-356.

[52] Vázquez, R.; Díaz, A.M. y Rodríguez, I.A. (1997): "Calidad de Servicio y Satisfacción del Consumidor: Un Enfoque Multidimensional para Empresas de Supermercados", ICE Tribuna de Economía, N. ${ }^{\circ} 763$, pp. 120-137.

[53] Zeithaml, V.A. (1988): "Consumer Perceptions of Price, Quality, and Value: A Means-End Model and Synthesis of Evidence", Journal of Marketing, Vol. 52, julio.

[54] Zeithaml, V.A.; Parasuraman, A. y Berry, L.L. (1985): "Problems and Strategies in Services Marketing", Journal of Marketing, Vol. 49, abril, pp. 33-46.

[55] Zeithaml, V.E.; Parasuraman, A. Berry, L.L. (1990): Delivering Quality Service: Balancing Customer Perceptions and Expectations, Nueva York: The Free Press. 